\title{
Electrolyte Comparison for Li-Metal Anodes with Cryo-Laser PFIB
}

Katherine Jungjohann ${ }^{1}$, Laura Merrill ${ }^{1}$, Renae Gannon ${ }^{2}$, Steven Randolph ${ }^{3}$, Daniel Long ${ }^{4}$ and Katharine Harrison $^{1}$

${ }^{1}$ Sandia National Laboratories, United States, ${ }^{2}$ University of Oregon, United States, ${ }^{3}$ Thermo Fisher Scientific, United States, ${ }^{4}$ Sandia National Laboratories, Albuquerque, New Mexico, United States

Insights into the interfacial reactions between lithium metal and volatile electrolytes provide the understanding needed to engineer lithium-metal batteries for high-energy density rechargeable energy storage. The integration of lithium-metal anodes into a rechargeable battery will provide significant gains in storage capacity and energy density over graphite electrodes used in lithium-ion batteries (LIB), but the uncontrolled interfacial reactions between lithium metal and common LIB electrolytes cause steady losses in performance due to uncontrolled solid electrolyte interphase (SEI) formation and Li-metal consumption. The SEI forms when electron transfer occurs at the surface of the Li-metal electrode, causing breakdown of the electrolyte into lithium containing species. Ideally these reactions create a passivating layer on the $\mathrm{Li}$ metal to prevent further reaction (to conserve the $\mathrm{Li}$ inventory) and to serve as a $\mathrm{Li}$-ion conductor. Self discharge is caused by an insufficiently passivating SEI to prevent the Li metal oxidation by the electrolyte. To produce a commercial grade Li-metal anode for rechargeable energy storage, a high performance SEI is required, with properties defined by the electrolyte and current density for Li metal plating and stripping.

Characterization of the SEI on Li-metal anodes is complicated by the solid-liquid interface that is buried within a stack of disparate materials. Commonly, small-scale battery performance testing is evaluated using coin cells, which comprises a stack of a metal current collector, anode, polymer separator filled with electrolyte, cathode, and another current collector that are held together with washers and a spring within a hermetically-sealed stainless steel casing. In general, characterization of these components requires the coin cell to be disassembled for analysis, which destroys the structures at the solid-liquid interfaces. Building on cryogenic electron microscopy techniques [1-2], we have shown that the complete battery stack can be visualized using a Laser Plasma Focused Ion Beam (Laser PFIB) as the coin cell is held at temperatures below $-100{ }^{\circ} \mathrm{C}$ [3]. Using this method, we have evaluated various electrolyte chemistries to understand the performance and thickness differences in the SEI evolved during cycling Li-metal half cells.

Commercial carbonate-based LIB electrolytes were compared to high-performance ether-based electrolytes for Li-metal anodes. The amount of evolved SEI and remaining Li inventory in the first $\mathrm{Li}$ plating and stripping cycles were evaluated, and after the $101^{\text {st }}$ plating cycle. These cross-sectional images from cryogenic analysis in the Laser PFIB provide insights on the relationships between the working and counter electrodes, interaction of the SEI with the polymer separator, and variations in the failure modes at the system level (Figure 1). These insights on Li-metal anode performance in varied electrolytes will be discussed along with the methods for obtaining clean cross-sectional images and energy dispersive $\mathrm{x}$ ray spectroscopy maps of the cryogenically frozen intact battery stacks using femtosecond laser ablation with the Thermo Fisher Scientific Laser PFIB [4-5]. 


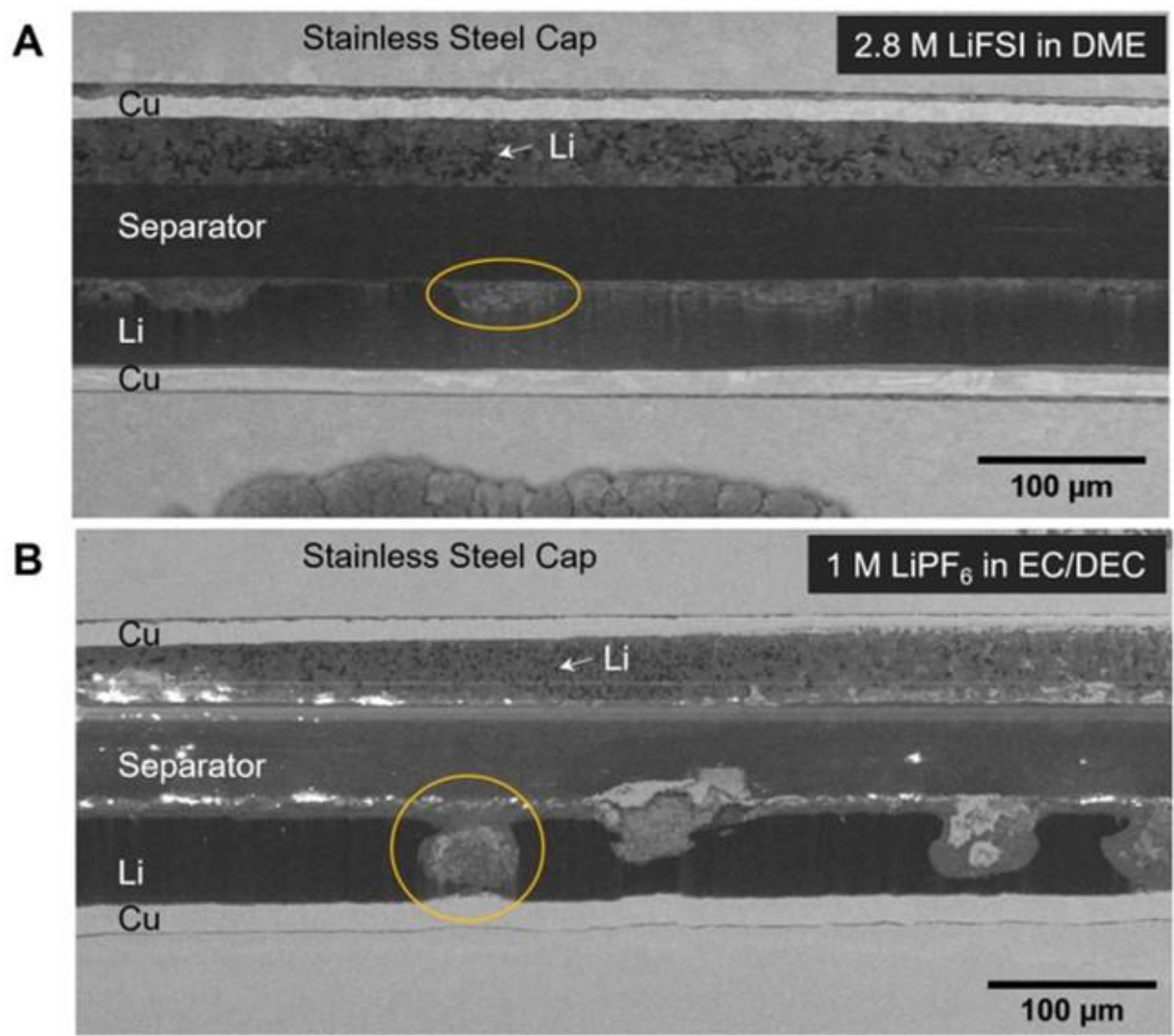

Figure 1. Figure 1. Scanning electron micrograph after cryo-Laser PFIB sectioning of intact coin cell battery stacks of a Li metal plated (top) vs. a Li metal counter electrode (bottom) after the 1st Li plating step at $0.47 \mathrm{~mA} / \mathrm{cm} 2$ in a) $2.8 \mathrm{M}$ lithium bis(fluorosulfulfonyl)imide in dimethoxyethane (LiFSI in DME), and b) $1 \mathrm{M}$ lithium hexafluorophosphate in ethylene carbonate and diethyl carbonate (LiPF6 in EC/DEC) with two Celgard 2325 separators. Differences in the electrodeposited Li grain size can be seen with the white arrows, and the different removal depths of the Li counter electrode can be seen in the yellow circles. SEI and electrolyte fills in spaces between the Li grains in the electrodeposited film.

\section{References}

[1] Y Li et al., Science 358 (2017), p. 506.

[2] MJ Zachman et al., Nature 560 (2018), p. 345.

[3] K Jungjohann et al., Microscopy and Microanalysis 26, S2 (2020), p. 1652.

[4] MP Echlin et al., Materials Characterization 100 (2015), p. 1.

[5] This work was funded by Sandia National Laboratories' Laboratory Directed Research and Development program. It was performed, in part, at the Center for Integrated Nanotechnologies, an Office of Science User Facility operated for the U.S. Department of Energy (DOE) Office of Science. Sandia National Laboratories is a multi-mission laboratory managed and operated by National Technology and Engineering Solutions of Sandia, LLC., a wholly owned subsidiary of Honeywell International, Inc., for the U.S. DOE's National Nuclear Security Administration under contract DE-NA-0003525. The views expressed in the article do not necessarily represent the views of the U.S. DOE or the United States Government. 